\title{
La Alameda. Espacio de libertad y discriminación
}

\author{
Efrén Sandoval Hernández
}

\section{Adela Díaz MeléndeZ, 2009 \\ Migración indígena y apropiación del espacio público en Monterrey. El caso de la Alameda \\ Centro de Estudios Históricos de la Universidad \\ de Monterrey, Centro de Investigaciones y Estudios \\ Superiores en Antropología Social, Facultad de Filosofía \\ y Letras de la Universidad Autónoma de Nuevo León, \\ Monterrey, México, 235 pp.}
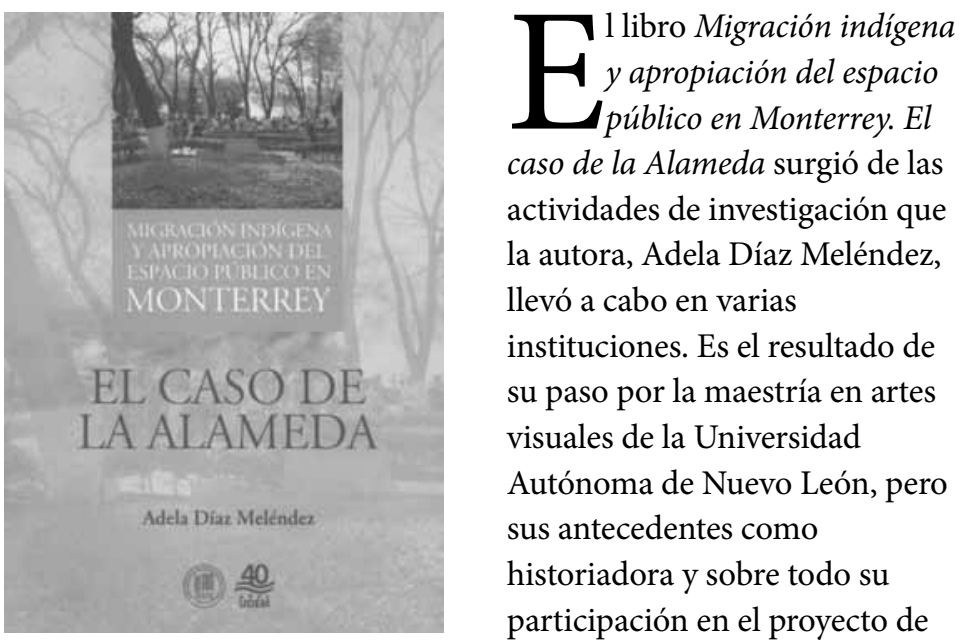
caso de la Alameda surgió de las actividades de investigación que la autora, Adela Díaz Meléndez, llevó a cabo en varias instituciones. Es el resultado de su paso por la maestría en artes visuales de la Universidad Autónoma de Nuevo León, pero sus antecedentes como historiadora y sobre todo su participación en el proyecto de

La Alameda. A Place of Freedom and Discrimination

Efrén Sandoval Hernández: Centro de Investigaciones y Estudios Superiores en Antropología Social-Programa Noreste, Monterrey, Nuevo León, México esandoval@ciesas.edu.mx

Desacatos, núm. 39, mayo-agosto 2012, pp. 195-200 investigación "Migrantes indígenas del área metropolitana de Monterrey" - patrocinado por el Consejo Nacional de Ciencia y Tecnología (Conacyt) y dirigido por Séverine Durin, del Centro de Investigaciones y Estudios Superiores en Antropología Social (CIESAS)-Programa Noreste- fueron fundamentales para la realización del estudio. En ese proyecto Adela Díaz

Meléndez desarrolló el caso de la Alameda Mariano Escobedo, un parque del centro de la ciudad de Monterrey, que hace varias décadas era visitado por las elites locales que en algún momento prefirieron vivir en los alrededores de la plaza, alejados 
del bullicio de las fábricas. Este espacio es estigmatizado ahora como uno de los lugares más populares, inseguros y desagradables de la ciudad. La Alameda ya no aparece en las postales de Monterrey y está fuera de cualquier guía turística de la ciudad. Lugar negado, la Alameda es hoy un espacio ocupado por miles de jóvenes, indígenas en su mayoría, que viven ahí la experiencia de ser jóvenes y libres, al menos durante el fin de semana.

\section{CONTENIDO DE LA OBRA}

El libro se plantea demostrar que la Alameda es un espacio

\section{estratégico de encuentro entre}

los indígenas migrantes, en especial para las mujeres que laboran como empleadas domésticas "puertas adentro". Desde el planteamiento de este objetivo, la autora nos presenta a las empleadas domésticas como actores sociales que realizan estrategias. La hipótesis es que la plaza de la Alameda, en el centro de Monterrey, es sede de relaciones y prácticas sociales que permiten a los migrantes recrear y ampliar sus redes sociales en su estancia en la ciudad. De esta manera, la Alameda se convierte en un espacio vivido y practicado, lo que le brinda un carácter nodal y la vuelve "estación" en la ciudad y en el proceso migratorio. Ante la ausencia de espacios propios de las migrantes indígenas que laboran como empleadas domésticas en la ciudad, se da un proceso de apropiación del espacio urbano público. ¿Por qué sucede esto? ¿Qué espacio corresponde tradicionalmente a una empleada doméstica? ¿El cuarto de servicio - que a veces merecería ser llamado lavandería con cama-? El libro demuestra que estas mujeres se apropian de un espacio público en el que se construyen como actores étnicamente diferenciados y sobre el que ejercen un control cultural respecto de su uso.

La autora argumenta que la Alameda es un espacio de acuerdo con tres perspectivas correlacionadas: a) espacio social y construido; b) espacio practicado, y c) espacio apropiado. El contenido del libro nos da bastantes ejemplos de cómo la Alameda se constituye a través de redes sociales, se practica a partir de una funcionalidad cotidiana y se apropia por medio de ciertas prácticas propias de grupos étnicos, de género o de edad. Entonces la Alameda no es sólo una plaza, es un espacio.

En la introducción se presenta un estado de la cuestión sobre redes sociales, migrantes indígenas en las ciudades - se destacan fenómenos similares en ciudades de otros países- y espacios públicos. Además, se describe la metodología de la investigación, en la que sobresale la observación participante y la escritura de diarios de campo. Se reflexiona sobre las dificultades para obtener información mediante preguntas directas y las ventajas de convertirse en uno más dentro del espacio practicado por los informantes. La autora comenta que cuando se hizo acompañar por alguien más durante algunos fines de semana al inicio del trabajo de campo: "el hecho de ser dos personas nos permitió un mayor disfrute de la Alameda, realizar actividades (como comer alguna fruta, tomar algún refresco o café) similares a las comunes de cualquier pareja de mujeres migrantes y por lo tanto 'ser' parte del espacio y ser vistas ahí por los propios migrantes". Creo que es una buena lección para los muchos encuestadores que cada fin de semana merodean la Alameda de Monterrey.

En el primer capítulo se habla de la migración indígena a Monterrey. Se proporcionan datos estadísticos y se hace un perfil de los y las indígenas que viven en esta ciudad y de aquellas con las que la autora tuvo interacción en la Alameda.

Además, se hace una caracterización del empleo doméstico en Monterrey. De acuerdo con la Ley Federal del Trabajo, este tipo de empleo es considerado en la categoría de "trabajos especiales", que se caracterizan por tener el mínimo de derechos y beneficios, rubro 
en el que en todo caso

los patrones deben tener

obligaciones especiales. La autora

destaca que las empleadas "de quedada" o "planta" realizan faenas diarias de hasta 12 horas, en las que sus responsabilidades no son muy diferentes a las que han aprendido desde niñas, como cuidar a niños pequeños o bebés, limpiar la casa, hacer la comida:

A pesar de que el empleo
doméstico es considerado
como "especial" y por lo tanto
sin obligaciones laborales
"ordinarias", los empleadores
cotizan y tabulan a las jóvenes
desde distintos aspectos:
la experiencia, las
responsabilidades y
habilidades en el trabajo.

Estas mujeres que trabajan como empleadas "de quedada" no cuentan con espacios propios de vivienda familiar, de descanso, ni de contacto diario con sus familiares y paisanos. Por ello, las jóvenes acuden en sus días de descanso al parque público: la Alameda Mariano Escobedo. Un sitio que antaño fue visitado por las elites regiomontanas, que después pasó a ser un lugar para el paseo para las familias de clase media y que ahora es usado por diferentes sectores o grupos sociales, algunos de los cuales acuden entre semana y otros durante el fin de semana. El segundo capítulo nos muestra cómo la Alameda sirve de soporte para los migrantes de la Huasteca.
La autora expone casos de migrantes de los poblados de Oxtomal y Huautla, en Hidalgo, y nos presenta la Alameda como un nodo, un "lugar de parada". Nos da información sobre su ubicación geográfica, las rutas de transporte colectivo que operan ahí, su cercanía con la terminal de autobuses y los diferentes servicios que se proporcionan a los migrantes, como el cuidado de maletas o guardería.

En el tercer capítulo la Alameda es expuesta por la autora como un espacio practicado - actor que practi$\mathrm{ca}-$, en el que se realizan actividades recreativas, en el que tiene lugar el encuentro entre los del mismo grupo, con los "regiomontanos": el comerciante, el policía, el encuestador, el bolero, el mesero. Pero la Alameda también es un "espacio estigmatizado", y a través de esos estigmas la autora muestra un actor social que está inmerso en una relación de desigualdad con "otro". Las empleadas domésticas son estigmatizadas por los antiguos usuarios de la plaza, los que han estado ahí desde mucho tiempo antes, lo mismo que por los medios de comunicación. El cuarto capítulo se ocupa de la migración femenina y las relaciones de género, en el que las empleadas domésticas son vistas como un actor que siente, que es individuo, persona. Adela Díaz Meléndez nos habla de la
Alameda como un espacio para ser joven, para el coqueteo, el noviazgo, el embarazo, el matrimonio, para organizar el regreso al pueblo.

\section{¿QUÉ ES LA ALAMEDA SEGÚN ESTE LIBRO?}

Pero, ¿qué es la Alameda según este libro? Es un espacio estratégico de encuentro, para descansar, para relacionarse con los parientes, amigos, paisanos y conversar en la lengua materna. Es un espacio de reunión, socialización y consolidación de redes sociales. Es un lugar antropológico en cuanto que encrucijada, donde los actores sociales se cruzan, se encuentran y se reúnen. Es un espacio construido por las relaciones y prácticas sociales ahí verificados. Los actores usan, practican y definen el espacio, lo espacializan en función de sus maneras de cumplir actividades y rutinas propias, dotándole de significados $\mathrm{y}$ distintas formas espaciales donde hacen intersección los encuentros sociales: es estación y lugar de parada. La Alameda es un nodo urbano, es un elemento que los migrantes han hecho suyo desde finales de la década de 1980. Antes era un lugar ajeno y que "pertenecía" - era identificado y practicado- a la sociedad hegemónica o dominante. Hoy es lugar de degradación, de comercio informal: "un antes familiar y 
tranquilo y un ahora de migrantes, suciedad y peligro".

La Alameda también es la central de autobuses, el local para dejar maletas, la caseta telefónica, el tablero con anuncios de "se solicita muchacha", la disco SAZ, el Bananas, el Versalles, el Hotel Alameda, el Mercado

Fundadores, el Cine Cuauhtémoc, la estética, los videojuegos de las chispas, el Pollo Loco, el lugar para comprar ropa, para comer, el Banco Azteca, el Elektra, es un nodo que vincula todos estos espacios, es el lugar para intercambiar información: “¿Cuánto te pagan?, ¿cómo te tratan?, ¿te van a pagar aguinaldo?, ¿te toca cuidar niños?, ¿vas a ir al pueblo?”. Es un lugar privilegiado en "la ruta de la distracción", paseo o compras. Es un espacio público que los fines de semana funciona como privado en tanto que ahí se llevan a cabo actividades propias e íntimas, como las reuniones familiares lejos del control de los empleadores: es la sala, es el comedor, la casa que no se tiene en Monterrey y que jamás se tendrá a falta de prestaciones laborales. La Alameda es espacio privado en contraposición con la casa de la empleadora, que es espacio ajeno, incluido el cuarto de servicio al que cualquier miembro de la familia puede entrar sin tocar la puerta. La Alameda es espacio para la toma de decisiones y acciones tan importantes como la de elegir una manera de ser joven, de ser mujer u hombre, de ser hija, de ser amiga, prima, madre.

La Alameda es a su vez el espacio para vestir la prenda prohibida en el pueblo, para coquetear, para sentirse bonita, para ser galán, para el enamoramiento, el primer beso, el primer faje, la primera pasión. La Alameda es espacio de opinión, de negociación, en un mundo de "contratos de adhesión, de contratos determinados unilateralmente por la parte empleadora con poca o ninguna discusión posible sobre sus cláusulas". Para muchas mujeres es "el respiro de la larga jornada de trabajo con niños, de preparar alimentos y limpiar la casa de la patrona”. Es el lugar para dejar de ser "la empleada", la muchacha, la chacha, para ser Claudia, Nicolasa, Juanita, Lucía, Rosa, Maribel, Azalea, Gloria, Mayela, Rosy, Susana, Ignacia, Ana, Francisca, la prima, la hermana, la paisana y, por qué no, la colega. Es lugar para compartir un origen, expectativas, el gusto por el zacahuil, la tortilla de maíz "de verdad". Es el lugar para aprender a vivir en la ciudad pero también para aprender zapoteco y enseñar náhuatl.

La Alameda es asimismo el lugar para el estigma, y como tal, es un lugar abandonado: "Abandonan los regios la Alameda", dice el encabezado periodístico. Es un lugar arrebatado y de arrebatos, "invadido", quitado, degradado, tomado por "los de fuera", es espacio ruidoso, feo, sucio, peligroso, problemático, inseguro, lugar para "gatas" y "albañiles", es Ciudad Gática, Alamegata, es un lugar para "gatear", donde vas y sales rasguñado, donde se comen whiskas, es un zoológico, miau, miau. Es el consulado o la embajada de San Luis, Tamazunchale Place, Hermana República de San Luis, lugar de oaxaquitas. Es Nacalameda, la Alamierda, lugar de los nacos, de las Marías, es el templo del mal gusto, el lugar de los crímenes, los infanticidios, los abandonos de bebés, los abortos y los embarazos no deseados: "Da a luz y estrangula bebé", "Le confiesa ser casado; ella estrangula a bebé", "El lado oscuro de la maternidad". Es el lugar en donde las empleadas domésticas "se salen de control".

Pero la Alameda también es el lugar para seguir viviendo en el pueblo. Ahí, allá, reciben noticias de la Alameda: "ya tiene novio", "salió embarazada", "cambió de patrona", "se porta muy bien en Monterrey”. A través del rumor y el chisme, el pueblo ejerce cierto "control" en la Alameda. Esta plaza también es una ventana para mirar desde el pueblo de origen, es un nodo para la prolongación de instituciones culturales locales. Ahí se reproducen las formas de hacer el matrimonio, de convertirse en 
adulto. En la Alameda la joven se entera de que ha sido pedida, desde ahí se organizan las quinceañeras, aun cuando tengan 18 años. Es un lugar para el cambio, para tener noviazgos de manera diferente, para casarse más tarde. A través de la práctica de la Alameda se van modificando y recreando instituciones sociales y culturales muy importantes para el lugar de origen.

Tomando en cuenta todo lo que la Alameda respresenta, podemos resumir que se convierte en un lugar de libertad, como la cocina para las esclavas africanas del Caribe mencionadas por Mintz (2003): como ese único espacio en donde se puede crear y recrear la cultura, los gustos, los saberes, en donde se puede ser. $\mathrm{Al}$ mismo tiempo, la Alameda es un lugar discriminado, es la discriminación vuelta objeto. Esa plaza se convierte en el objeto de discriminación para disimular la discriminación a las personas. La Alameda es un cristal para mirar la relación entre migrantes y la sociedad receptora.

\section{¿POR QUÉ ES IMPORTANTE LA PUBLICACIÓN DE ESTE LIBRO?}

\section{La publicación de Migración} indígena y apropiación del espacio público en Monterrey. El caso de la Alameda, de Adela Díaz Meléndez, es importante por las paradojas y las contradicciones que evidencia. Primero, la Alameda es un lugar de libertad para las jóvenes trabajadoras domésticas, una libertad acotada a un espacio que de por sí es considerado menor, inferior, como secundario, periférico. La Alameda, contradictoria y dialécticamente, da libertad al mismo tiempo que reitera que "nosotros", en este caso las empleadas domésticas, sólo tenemos derecho a usar este espacio de esta manera y en este tiempo determinado. Así confirmamos nuestra posición en esta sociedad que de diferentes maneras nos recuerda que no podemos ir más allá. ¿Será la diferencia principal respecto del caso de Guadalajara mencionado en el libro? ¿Será que al menos las empleadas domésticas de San Pedro Garza García, en el área metropolitana de Monterrey, no han osado apropiarse de un espacio cercano a su lugar de trabajo o que forma parte de la zona residencial en la que trabajan? ¿Será a cambio de esa distancia que se les concede este espacio y este tiempo de libertad?

En segundo lugar, la autora proporciona ejemplos de jóvenes $\mathrm{y}$ familias que han emigrado durante generaciones, cómo consiguen trabajo, qué labores desempeñan, en qué condiciones las realizan, cómo se relacionan con paisanos y parientes, cómo se comunican, cómo aprenden y se hacen novios, etcétera. En cada ejemplo la autora da el nombre de la persona en cuestión, nos dice cuál es su origen y su edad. Este último dato llamó mi atención. Aunque la edad de las entrevistadas era de 16,18 o 20 años, y la relatora es una mujer de 30 años, su experiencia laboral en Monterrey, su primer trabajo, su primera migración fue a los 12, 13 o 14 años. Se trata de trabajo infantil: tenemos a niñas trabajando en nuestras casas.

A mediados de 2009 hubo una campaña de escandalización en los periódicos de Monterrey: había menores de edad prostituyéndose en un table dance. Alborto mayúsculo para una sociedad acostumbrada a mirarse a sí misma sólo a través del filtro tendencioso de los medios de comunicación. Nadie se escandaliza por tener en su casa trabajando informalmente a una menor de edad, por no darle prestaciones, por despedirla cuando se embaraza, pero sí es causa de escándalo que los indios se diviertan, se reúnan, platiquen, sonrían, se aventuren, se arriesguen, que sean jóvenes y se atrevan, simplemente, a ser, aunque sea en un espacio marginal. La mujer india, niña o joven, que pretende ser libre es discriminada y abandonada a la suerte no sólo de un mundo machista, violento y abusivo, sino hasta de políticos paranoicos que las creen criminales en potencia, como el alcalde de San Pedro Garza García que tiene la intención 
de hacer un censo de empleadas domésticas bajo la sospecha de que pasan información y coparticipan con los secuestradores.

En tercer término, este libro habla de otras contradicciones. Una de ellas es la relación laboral. Ya mencioné que en muchos casos se trata de trabajo infantil, pero hay que subrayar que es una relación laboral determinada casi en su totalidad por el patrón o patrona. En una relación así la posibilidad de negociar del empleado es casi nula, de manera que eso que algunos llaman "mañas", como no limpiar por aquí, ganar tiempo por allá, el aprovecharse de ciertos recursos de la casa en que se trabaja, vistos desde el punto de vista del empleado, son tal vez las únicas vías disponibles para sacar algún provecho de una relación totalmente desventajosa. Más aun, en una sociedad liberal porfirista, como han definido Zúñiga y Contreras (1998) a la sociedad regiomontana en su estudio sobre la pobreza. Es totalmente contradictorio que se despotrique contra aquellos que no pagan impuestos cuando se evade la mínima responsabilidad de dar derechos a quien labora en nuestra propia casa.

Por último, este libro nos ayuda, y tengo la esperanza de que ayude a muchos de sus lectores, a mirarnos a nosotros mismos sobre todo por lo que dice sobre los que no vamos a la Alameda. Este libro habla de la relación entre "ellos" y "nosotros", donde "ellos" no sólo son diferentes, sino desiguales. Este libro debe enseñarnos a vernos a nosotros mismos no sólo a través de investigaciones como ésta o de lugares como la Alameda.

Debemos aprender a ver esta realidad en nuestra vida cotidiana, en nuestra relación con los empleados domésticos, con los "foráneos", con los indios $\mathrm{y}$ con los diferentes - porque este libro se refiere a todo eso cuando nos habla de las empleadas domésticas-. Debemos preguntarnos, ¿cuándo, en qué momento nos acostumbramos o aprendimos que es natural mantener una relación así con un empleado? ¿Cuándo aprendí que a la servidumbre no se le saluda? ¿Cuándo aprendí que un indio no puede tener derechos? ¿A qué hora me dijeron que el progreso no tiene por qué incluirlos a ellos? ¿Cuándo aprendí que es aceptable tener a alguien a mi servicio personal? ¿Será todo esto uno de los rasgos más profundos de nuestra cultura?

\section{Referencias}

Mintz, Sidney W., 2003, Sabor a comida, sabor a libertad. Incursiones en la comida, la cultura y el pasado, Centro de Investigaciones y Estudios Superiores en Antropología Social, Ediciones de la Reina Roja, Consejo Nacional para la Cultura y las Artes, México.

Zúñiga, Víctor y Óscar Contreras, 1998, "La pobreza en Monterrey", en Luis Lauro Garza (coord.), Nuevo León hoy, La Jornada Ediciones, Universidad Autónoma de Nuevo León, México. 